\title{
The Influence of Executives' Gender Differences in Listed Companies on Investment and Financing Decisions
}

\author{
Liu Xin-hua ${ }^{1, a}$, Fu Meng-ting ${ }^{1, b}$ andLv Sa-sa ${ }^{1, c}$ \\ ${ }^{1}$ School of Donlinks Economics and Management, University of Science and Technology Beijing, \\ 100083 China \\ aliuxh@management.ustb.edu.cn, bhero569@163.com, 'Ivsasa513029@163.com
}

\begin{abstract}
This paperuse the private enterprises which listed in A-shares from 2009 to 2013 as samples to examine the influence of executives' (chairman, CEO, CFO) gender differences on investment decisions and financing preference. The results show: the impact of gender on the company's investment efficiency and M \& A decision-making is not obvious, but if the listed company has female chairman, CEO or CFO, it will significantly slow the growth rate of company size and reduce the size of the company's external financing, a further selection of external financing shows female executives will be more inclined to choose equity financing, thereby reducing the company's debt to equity ratio.This study gives suggestions on the selection of new executives and optimizing the structure of the senior management team.
\end{abstract}

Keywords: executives'gender difference, private enterprises, investment decision-making, financing preference.

\section{高管性别差异对上市公司投融资决策的影响研究 \\ 刘欣华 ${ }^{1, a}$, 傅梦婷 ${ }^{1, b}$, 吕萨萨 ${ }^{1, c}$ \\ ${ }^{1}$ 北京科技大学东凌经济管理学院，北京，中国}

aliuxh@management.ustb.edu.cn, bhero569@163.com, 'Ivsasa513029@163.com

中文摘要.本文以2009-2013年A股民营上市 公司为样本，实证检验了高管（董事长、 $\mathrm{CEO}$ 和 $\mathrm{CFO}$ ) 的性别差异与公司投资决策和 融资偏好的关系。研究表明: 高管性别对公 司投资效率和并购决策的影响并不明显, 但 董事长、CEO和CFO中含有女性成员的公司 会显著减缓公司规模的增长速度, 也会明显 降低公司的外部融资规模, 在选择外部融资 方式时, 存在女性高管的上市公司更加倾向 于选择股权融资, 从而降低公司的债股比 例。本研究对上市公司选拔高管人员、优化 高管团队的性别结构有一定的借鉴意义。
关键词:高管性别差异; 民营企业; 投资决 策; 融资偏好

\section{1. 引言}

随着女性社会地位的提高, 其在公司管 理中的作用日益凸显, 国内外研究女性董事 及高管与公司表现关系的文献也随之兴起。 已有的研究主要集中于探讨女性高管对公 司业绩与价值、薪酬差距及财务行为等的影 响, 对高管性别差异与公司投资决策及外部 融资偏好的关系的研究甚少。高管性别差异 通常意味着管理风格和决策偏好不同, 大部 
分女性高管拥有丰富的经验、宽阔的眼界和 较高层次的社会关系网络, 能够选择合理的 决策方案使公司更好地发展。值得关注的 是, 我国上市公司高管团队中的女性比例仍 然很低, 其中董事会中的女性成员占比不到 $10 \%$, 首席级别的女性高管更加风毛麟角。

本文通过探讨我国民营上市公司的高 管性别差异对其投资决策与融资偏好的影 响, 希望公司在聘任高管时能够摒弃性别歧 视, 客观公正的看待高管性别与投融资决策 之间的关系, 优化高管团队的性别结构, 从 而改善公司业绩。

\section{2. 文献回顾与研究设计}

\section{1 高管性别差异与上市公司投资行为}

男女高管对公司的影响主要表现在性 别和与生俱来的性格特质的不同。 Zuckerman（1995）认为女性高管和男性有 不同的特质, 比如相对保守和谨慎、不过度 自信等。Jiekun Huang \& Darren J. Kisgen (2013) 研究发现, 相比于女性高管, 男性 高管会采取更多的并购行为、发行更多的公 司债券, 男性高管任职的公司并购更多, 发 行债券的公告收益更低。此外, 女性高管通 常会对公司有一个更宽泛的盈利预测, 并倾 向于更早的实施股票期权, 这一结论与 Zuckerman(1994) 的观点基本一致。Maurice Levi \& Kai Li et al. (2014) 认为女性董 事会影响公司投资行为, 以1997-2009年 S\&P1500的上市公司为研究样本, 结果发现 女性董事会采取更少的并购决策, 相比于男 性董事, 女性董事会在并购中支付更低的并 购溢价, 研究结果支持了女性董事会通过并 购行为更好的为公司股东创造价值的假设。

Barber \& Odean (2001) 的研究认为, 在与 金融相关的任务如投资行为上, 男性表现出 更高程度的过度自信。赵冰梅、吴会敏

（2013）从固定资产投资、无形资产投资、 长期股权投资三个方面进行考察, 结论是优 秀的女性高管和男性高管一样可以为上市 公司做出正确的、适当的投资决策。基于上 述分析, 本文认为男性高管通常会表现出更 高程度的过度自信, 相比之下, 女性高管由
于本身的细心和谨慎, 在投资决策过程中可 能会表现出保守的一面, 因此提出假设:

H1：相比于男性高管，女性高管会减 少公司的过度投资, 加重投资不足, 并采取 更少的并购决策。

\section{2 高管性别差异与上市公司融资偏好}

按照 “融资顺序理论 (Pecking financial order theory）”, 公司的融资决策是由各种 因素如控制权分散程度和财务成本高低决 定, 即首先会选择无成本的内部融资方式, 其次选择交易成本和风险较低的债务融资 方式, 最后才会选择股权融资的方式。目前 直接针对高管性别差异与公司融资偏好关 系的研究并不多, 研究结论表明, 相比于男 性的过度自信, 女性通常表现出更高的风险 厌恶，从而倾向于选择风险较低的投资组 合。Graham \& Harvey（2013）采用调查问 卷的方式对美国CEO的心理特征与公司行 为进行了分析考察, 研究发现CEO的风险厌 恶程度会影响公司的融资决策。李世刚

(2014) 实证检验了上市公司女性高管对管 理者过度自信、上市公司融资偏好的影响, 结果表明女性高管能显著降低上市公司过 度自信的概率, 降低上市公司投资一现金流 的敏感性, 降低内部融资偏好; 在控制女性 高管影响后, 管理者过度自信增强了内部融 资偏好。因此本文认为相比于男性高管, 女 性高管在制定融资决策时, 通常会选择风险 更小、成本更低的融资途径。因此提出假设:

H2：相比于男性高管，女性高管会选 择更少的外部融资, 如果选择外部融资方 式，更倾向于选择债权融资。

\section{3. 研究设计}

\section{1样本选取}

本文以2009-2013年A股民营上市公司 为样本, 并按如下标准进行数据篮选: (1) 模型中多数变量的计算需使用前一年数据, 故剔除当年上市的公司; (2) 剔除ST类公 司、金融行业和数据不完整的公司以及数据 异常观测值。最终得到1316家上市公司, 5044 个观测值。数据来源于RESSET数据 库, 并经过计算及手工篮选得到。 


\section{2变量界定}

\subsection{1 高管界定}

迄今为止, 学术界对于企业管理者并没 有形成统一的认识, 不同研究文献中的管理 者所包含的内容不尽相同。借鉴前人的研究, 考虑到并非所有的高管都会对公司的投资决 策和融资偏好产生重大影响, 本文界定的公 司高管只包括董事长、CEO和CFO。

\subsection{2 投资决策与融资偏好的衡量}

本文从投资效率、并购及公司规模增长 三个方面衡量投资决策。其中, 并购行为采 用虚拟变量, 公司规模增长用总资产增长率 衡量。投资效率借鉴 Richardson( 2006)的方 法衡量, 具体模型如下:

Investment $_{t}={ }_{0}+{ }_{1}$ Salesgrowth $_{t 1}+{ }_{2}$ Age $_{t 1}$ $+{ }_{3}$ Size $_{t 1}+{ }_{4}$ Cash $_{t 1}+{ }_{5}$ Lev $_{t 1}+{ }_{6}$ Investment $_{t 1}$ + YearDummy + InduDummy ${ }_{i, t}$

其中, Investment $t_{i, t}$ 为 $i$ 公司第 $t$ 年的总投 资, 即公司对外资本支出、收购支出和研究 开发支出再扣除资产处置收入; Salegrowth $_{i, t-1}$ 表示 $i$ 公司第 $t-1$ 年的销售收入 增长率; $A \mathrm{ge}_{i, t-1}$ 为公司年龄; $S i z e_{i, t-1}$ 为公 司规模, 即总资产的自然对数; $C a s h_{i, t-1}$ 、 $L e v_{i, t-1}$ 分别为公司现金持有量和资产负债 率; $R E T_{i t-1}$ 为公司股票 $t-1$ 年的回报率; Investment $_{i, t-1}$ 为 $i$ 公司 $t-1$ 年的总投资; YearDummy 和InduDummy 分别为年份和 行业的哑变量; $\varepsilon_{i, t}$ 为残差, $\varepsilon_{i, t}$ 为正则说明 投资过度，为负则说明投资不足。

探讨高管性别差异对外部融资偏好影 响的量化指标分两部分: 一是外部融资总 额, 包括股权融资, 也包括银行借款和应付 债券组成的债权融资; 二是外部融资结构, 采用债权股权比作为代理变量。

本文选取的控制变量包括两方面：一 是衡量管理层及董事会特征的变量, 包括管 理层平均薪酬 (其中管理层含RESSET数据 库中的所有管理者, 并非本文界定的高管)、 董事会规模、独立董事比例及女性董事比 例; 二是衡量公司特征的变量, 包括资产负
债率、资产收益率、净资产增长率及总资产。 主要变量的界定见表1:

表1 变量定义

\begin{tabular}{|c|c|c|}
\hline 变量符号 & 变量名称 & 变量定义 \\
\hline Inv & 投资效率 & 残差为正投资过度, 为负投资不足。 \\
\hline Acquisition & 并购 & 当年发生并购则为 1 , 否则为 0 \\
\hline Totassgrrt & 总资产增长率 & 当年总资产增长率 \\
\hline Finance & 外部融资 & Ln (银行借款+应付债券+发行总股数) \\
\hline LoanShare1 & 债权股权比1 & (银行借款+应付债券)/发行总股数 \\
\hline LoanShare2 & 债权股权比2 & (银行借款+应付债券)/流通股股数 \\
\hline Female & 高管性别 & $\begin{array}{l}\text { 董事长、 } \mathrm{CEO} \text { 和CFO中若有女性成员为 } \\
1 \text {, 否则为 } 0\end{array}$ \\
\hline Salary & 管理者薪酬 & 管理者的平均薪酬的自然对数 \\
\hline DirNum & 董事会规模 & 董事会成员人数 \\
\hline Gender & 董事会性别比例 & 女性董事占董事会总人数的比值 \\
\hline IndDirPct & 董事会独立性 & 独立董事占董事会总人数的比值 \\
\hline OwnCon5 & 股权集中度 & 前五大股东持股比例 \\
\hline Size & 公司规模 & 总资产的自然对数 \\
\hline Netgrowth & 净资产增长率 & $\begin{array}{l}\text { (期末净资产-期初净资产)/期初净资产 } \\
\text { *100\% }\end{array}$ \\
\hline ROA & 资产收益率 & 净利润/总资产*100\% \\
\hline
\end{tabular}

3.2.3投资决策与融资偏好的衡量 本文构建如下的多元回归模型 :

（1）投资决策模型

$$
\begin{aligned}
& \text { Inv }_{i, t}={ }_{0}+{ }_{1} \text { Female }_{i, t}+{ }_{2} \text { Inv }_{i, t 1}+{ }_{3} \text { Controls }_{i, t} 1 \\
& + \text { YearDummy }+ \text { InduDummy }+
\end{aligned}
$$

\section{Logit $\left._{\left(\text {Acquisition }_{i, t}\right.}\right)={ }_{0}+{ }_{1}$ Female $_{i, t}+$}

${ }_{2}$ Controls $_{i, t}+$ YearDummy + InduDummy $+{ }_{i, t}$

Totassgrrt $_{i, t}={ }_{0}+{ }_{1}$ Female $_{i, t}+{ }_{2}$ Controls $_{i, t}$ + YearDummy +InduDummy ${ }_{i, t}$

\section{（2）融资决策模型}

$$
\begin{aligned}
& \text { Finance }_{i, t}={ }_{0}+{ }_{1} \text { Female }_{i, t}+{ }_{2} \text { Controls }_{i, t} 1 \\
& + \text { YearDummy + InduDummy }+{ }_{i, t}
\end{aligned}
$$




\section{4. 实证分析}

\section{1描述性统计分析}

主要变量的描述性统计结果见表2-4。 由表2可以看出, 董事长、CEO和CFO中至 少有一名为女性的公司占样本 $34.4 \%$ 。结合 表3 可知, $\mathrm{CFO}$ 为女性的占比最大, 其次是 CEO, 女性任董事长的样本公司很少; 女性 高管比例基本上呈逐年增长的趋势, 说明越 来越多的女性加入到了公司高管团队, 女性 在公司治理等方面的能力得到了更高程度 的认可。从资产收益率、净资产收益率、总 资产收益率的均值和中位数可以看出, 绝大 部分公司都保持了较好的盈利和成长能力, 董事会规模平均约为 11 人, 其中独立董事和 女性董事占比总体偏低。前五大股东的持股 比例较高, 平均为 $55.8 \%, 23.6 \%$ 的公司在 样本年度内有并购行为。外部融资的自然对 数均值为 29.25 , 且Loanshare 1 和Loanshare 2 的平均值均低于 $50 \%$, 说明大部分公司在选 择外部融资时都会优先考虑股权融资。

进一步将样本公司按照董事长、CEO 和CFO中是否含有女性成员分组, 并进行样 本均值 T检验。由表 3 的分组检验结果可知, 相比于女性高管样本组的公司, 男性高管组 的公司规模更大、董事会中女性成员的占比 更低、管理层的平均工资更高、前五大股东 持股比例也更高。另外, 资产收益率和净资 产增长率的分组均值检验的 $\mathrm{T}$ 值均不显著, 表明男性高管在公司治理等方面的能力并 没有显著优于女性高管。投融资决策方面, 女性高管组的样本公司投资不足的情况更 严重, 在样本年度内有更少的并购行为, 在 公司制定融资决策时也更倾向于选择债权 股权比更低的融资结构。

表2全样本描述性统计

\begin{tabular}{|c|c|c|c|c|c|c|}
\hline 变量符号 & $\mathbf{N}$ & 均值 & 中位数 & 最小值 & 最大值 & 标准差 \\
\hline Female & 5044 & 0.344 & 0 & 0 & 1 & 0.475 \\
\hline DirNum & 5044 & 10.661 & 10 & 5 & 38 & 3.491 \\
\hline IndDirPct & 5044 & 0.357 & 0.333 & 0 & 0.833 & 0.090 \\
\hline Gender & 5044 & 0.109 & 0.100 & 0 & 0.714 & 0.104 \\
\hline Salary & 5044 & 12.086 & 12.097 & 9.327 & 15.035 & 0.668 \\
\hline ROA & 5044 & 0.062 & 0.058 & -7.285 & 5.074 & 0.211 \\
\hline Netgrowth & 5044 & 0.586 & 0.091 & -30.428 & 62.344 & 1.778 \\
\hline OwnCon5 & 5044 & 0.558 & 0.567 & 0.030 & 0.998 & 0.182 \\
\hline
\end{tabular}

\begin{tabular}{|c|c|c|c|c|c|c|}
\hline Size & 5017 & 21.069 & 21.007 & 10.842 & 25.056 & 1.073 \\
\hline Inv & 4809 & -0.205 & 0.544 & -38.509 & 12.659 & 6.004 \\
\hline Acquisition & 5044 & 0.236 & 0 & 0 & 1 & 0.424 \\
\hline Totassgrrt & 5041 & 0.261 & 0.115 & -0.966 & 10.735 & 0.612 \\
\hline Loanshare1 & 5035 & 0.409 & 0 & 0 & 1.182 & 0.499 \\
\hline Loanshare2 & 5035 & 0.493 & 0 & 0 & 2.200 & 0.612 \\
\hline Finance & 5035 & 29.249 & 19.998 & 17.399 & 67.461 & 12.688 \\
\hline
\end{tabular}

表3 女性高管公司与男性高管公司对比

\begin{tabular}{|c|c|c|c|c|c|c|c|}
\hline \multirow{2}{*}{$\begin{array}{c}\text { 变量符 } \\
\text { 号 }\end{array}$} & \multicolumn{3}{|c|}{ 女性高管组 } & \multicolumn{3}{c|}{ 男性高管组 } & \multirow{2}{*}{ 均值T } \\
\cline { 2 - 6 } & $\boldsymbol{N}$ & 均值 & 中位数 & $\boldsymbol{N}$ & 均值 & 中位数 & 检验 \\
\hline Size & 1724 & 20.97 & 20.913 & 3293 & 21.12 & 21.06 & $-4.69^{* * *}$ \\
\hline DirNum & 1736 & 10.76 & 10 & 3308 & 10.61 & 10 & 1.417 \\
\hline IndDirPct & 1736 & 0.356 & 0.333 & 3308 & .3577 & 0.333 & -.806 \\
\hline Gender & 1736 & 0.118 & 0.111 & 3308 & .1047 & 0.100 & $4.174^{* * *}$ \\
\hline Salary & 1736 & 12.04 & 12.050 & 3308 & 12.11 & 12.12 & $-3.82^{* * *}$ \\
\hline ROA & 1736 & 0.060 & 0.060 & 3308 & .0624 & 0.057 & -.338 \\
\hline Netgrowth & 1736 & 0.604 & 0.093 & 3308 & .5771 & 0.090 & .509 \\
\hline OwnCon5 & 1736 & 0.564 & 0.575 & 3308 & .5545 & 0.565 & $1.734^{*}$ \\
\hline Inv & 1622 & -0.40 & 0.550 & 3187 & -0.92 & 0.544 & $-1.795^{*}$ \\
\hline Acquisition & 1736 & 0.221 & 0 & 3308 & .2430 & 0 & $-1.738^{*}$ \\
\hline Totassgrrt & 1735 & 0.255 & 0.104 & 3306 & .2647 & 0.119 & -.541 \\
\hline Loanshare1 & 1736 & 0.431 & 0 & 3308 & .5240 & 0 & $-5.11^{* * *}$ \\
\hline Loanshare2 & 1733 & 0.432 & 0 & 3302 & .5249 & 0 & $-5.11^{* * *}$ \\
\hline Finance & 1733 & 27.99 & 19.707 & 3302 & 29.91 & 20.22 & $-5.13^{* * * 1}$ \\
\hline
\end{tabular}

表4 各年份女性高管分布

\begin{tabular}{|c|c|c|c|c|c|c|}
\hline 年份 & 2008年 & 2009年 & 2010年 & 2011年 & 2012年 & 合计 \\
\hline 女性董事长 & 13 & 14 & 13 & 15 & 15 & 70 \\
\hline 女性CEO & 32 & 51 & 77 & 85 & 89 & 334 \\
\hline 女性CFO & 155 & 200 & 296 & 366 & 414 & 1431 \\
\hline $\begin{array}{c}\text { 高管中至少 } \\
\text { 一人为女性 }\end{array}$ & 189 & 249 & 370 & 443 & 485 & 1736 \\
\hline
\end{tabular}

\section{2变量相关性检验}

从表 5 可见,各自变量之间的相关系数 绝对值均小于 0.3 , 本文回归模型中的自变 量之间不存在严重的多重共线性问题。

\section{3回归分析}

\subsection{1 高管性别差异与上市公司投资决策}

从表 6 可见，将投资效率分为投资过度 和投资不足两个样本组, 未控制年度和行业 变量时, 女性高管会加重上市公司的投资过 度和投资不足，对投资不足的影响更严重， 但在控制年度和行业变量后, 这种关系并不 显著。另外，女性高管与公司并购决策呈负 相关关系, 说明董事长、CEO和CFO中含有女 性成员倾向采取更少的并购, 这与假设一的 方向一致，但并不显著。总资产增长率与高 管性别的相关系数为 $-0.037 、-0.029$, 且在 $1 \%$ 和 $10 \%$ 的水平上显著，表明高管中含女性 会减缓公司规模的增长速度, 与女性高管比 男性高管更加谨慎的假说基本一致。管理层 的平均薪酬、大股东持股及净资产增长率可 
以抑制公司的无效率投资问题, 其中净资产 增长率能增加公司的并购行为、减缓公司规 模的增长速度。

上述回归结果只部分支持了研究假设 一, 说明董事长、CEO和CFO中是否含有女性 成员对公司的投资效率和并购决策的影响 不存在明显的差异。
4.3.2 高管性别差异与上市公司融资偏好

表7中，因变量为外部融资时，高管性 别在控制年份和行业变量前后的回归系数 分别为 -0.974 和 -0.900 , 且均在 $1 \%$ 的水平上 显著, 即高管性别变量每增加一个单位, 公 司的外部融资总额就平均减少 0.974 和 0.900个单位, 说明高管中含女性高管会显 著减少上市公司的外部融资。

表 5 相关系数矩阵

\begin{tabular}{|c|c|c|c|c|c|c|c|c|c|}
\hline & Female & DirNum & IndDirPct & Gender & Salary & ROA & Netgrowth & OwnCon5 & Size \\
\hline Female & 1 & & & & & & & & \\
\hline DirNum & 0.020 & 1 & & & & & & & \\
\hline IndDirPct & -0.011 & $-0.290^{* * * *}$ & 1 & & & & & & \\
\hline Gender & $0.059^{* * *}$ & $-0.112^{* * *}$ & $0.053^{* * *}$ & 1 & & & & & \\
\hline Salary & $-0.054^{* * *}$ & $-0.118^{* * *}$ & $0.046^{* * *}$ & $-0.030^{* * *}$ & 1 & & & & \\
\hline ROA & -0.005 & $-0.068^{* * *}$ & 0.018 & 0.021 & $0.096^{* * *}$ & 1 & & & \\
\hline Netgrowth & 0.007 & $-0.122^{* * *}$ & 0.013 & 0.013 & 0.017 & $0.136^{* * *}$ & 1 & & \\
\hline OwnCon5 & $0.024^{* * *}$ & $-0.292^{* * *}$ & $0.100^{* * *}$ & -0.005 & $0.136^{* * *}$ & $0.152^{* * *}$ & $0.161^{* * *}$ & 1 & 1 \\
\hline Size & $-0.067^{* * * *}$ & $0.075^{* * * *}$ & -0.019 & $-0.024^{* * *}$ & $0.481^{* * *}$ & $-0.071^{* * *}$ & -0.010 & -0.012 & 1 \\
\hline
\end{tabular}

注: $*, * *, * * *$ 分别表示在 $10 \%, 5 \%, 1 \%$ 的水平上显著

表6 高管性别差异与上市公司投资决策

\begin{tabular}{|c|c|c|c|c|c|c|c|c|}
\hline \multirow{3}{*}{$\begin{array}{l}\text { 变量符号 } \\
\text { Constant }\end{array}$} & \multicolumn{4}{|c|}{ 投资效率 } & \multirow{2}{*}{\multicolumn{2}{|c|}{$\begin{array}{c}\text { 并购 } \\
\text { 投资过度 }\end{array}$}} & \multirow{2}{*}{\multicolumn{2}{|c|}{$\begin{array}{c}\text { 总资产增长率 } \\
\text { 投资不足 }\end{array}$}} \\
\hline & \multicolumn{2}{|c|}{ 投资过度 } & \multicolumn{2}{|c|}{ 投资不足 } & & & & \\
\hline & $4.760^{* * * \pi}$ & $8.867^{* * * *}$ & $-15.204^{* * 4}$ & $-23.740^{* * *}$ & $-0.870^{* * 1}$ & $-1.288^{* \pi *}$ & $2.031^{* * x+1}$ & $1.465^{* * *}$ \\
\hline Female & $0.165^{* *}$ & 0.030 & $-1.140^{* * *=}$ & -0.655 & -0.014 & -0.008 & $-0.037^{* 3}$ & $-0.029^{*}$ \\
\hline DirNum & $0.068^{* * \pi}$ & 0.030 & $-0.283^{*}$ & $-0.161^{\prime \prime}$ & $0.003^{n}$ & 0.000 & -0.001 & -0.001 \\
\hline IndDirPct & -0.445 & -0.494 & -0.792 & -2.350 & -0.007 & 0.048 & -0.008 & 0.065 \\
\hline Gender & 0.439 & 0.201 & $-4.635^{* *}$ & $-3.608^{* *}$ & -0.022 & 0.015 & $-0.143^{*}$ & -0.102 \\
\hline Salary & $-0.109^{* * *}$ & $-0.185^{3 * \pi *}$ & $0.660^{*}$ & $0.852^{2 *}$ & -0.007 & 0.014 & $0.050^{* \pi *}$ & $0.069^{* * 7 * 4}$ \\
\hline ROA & 0.073 & 0.275 & $5.073^{* * *}$ & $6.886^{* * *}$ & 0.060 & 0.036 & $0.531^{* * *}$ & $0.507^{* * *}$ \\
\hline Netgrowth & $-0.051^{* \prime \prime}$ & -0.035 & $0.296^{* \pi}$ & 0.193 & 0.014 & 0.005 & -0.028 & $-0.031^{* n+4}$ \\
\hline OwnCon5 & $-1.545^{* * *}$ & $-1.225^{* * *}$ & $12.963^{* * *}$ & $10.218^{* * *}$ & -0.051 & $-0.075^{\text {** }}$ & $0.933^{* * *}$ & $0.934^{* * *}$ \\
\hline Size & $-0.074^{\pi n}$ & $-0.209^{n+\cdots}$ & -0.026 & 0.256 & 0.056 & $0.069^{n+1}$ & -0.137 & $-0.126^{*}$ \\
\hline Year & & Yes & & Yes & & Yes & & Yes \\
\hline Industry & & Yes & & Yes & & Yes & & Yes \\
\hline F值 & $24.76^{* * * *}$ & $15.39^{n+m}$ & $26.37^{\text {*** }}$ & $14.67^{* *}$ & $13.88^{* * * *}$ & $61.37^{* \pi \times 2}$ & $118.88^{* *}$ & $105.53^{* \pi}$ \\
\hline $\mathrm{N}$ & 3178 & 3178 & 1631 & 1631 & 5017 & 5017 & 5017 & 5017 \\
\hline Adj-R ${ }^{2}$ & 0.0631 & 0.2652 & 0.1229 & 0.2304 & 0.0226 & 0.1441 & 0.1746 & 0.2221 \\
\hline
\end{tabular}

注: $*, * *, * * *$ 分别表示在 $10 \%, 5 \%, 1 \%$ 的水平上显著 
表7 高管性别差异与上市公司融资偏好

\begin{tabular}{|c|c|c|c|c|c|c|}
\hline \multirow{2}{*}{ 变量符号 } & \multicolumn{2}{|c|}{ 外部融资 } & \multicolumn{2}{|c|}{ 债权股权比1 } & \multicolumn{2}{|c|}{ 债权股权比 2} \\
\hline & 投资过度 & 投资不足 & 投资过度 & 投资不足 & 投资过度 & 投资不足 \\
\hline Constant & $-92.631^{2 * 2 x}$ & $-93.056^{* N "}$ & -4.726 & $-4.741^{* m+}$ & -4.726 (") & $-4.742^{* * \pi *}$ \\
\hline Female & $-0.974^{* \pi}$ & $-0.900^{* * 1}$ & $-0.052^{* *}$ & $-0.047^{*}$ & $-0.052^{m+1}$ & $-0.047^{*}$ \\
\hline DirNum & $0.183^{* * * *}$ & $0.167^{* * * \pi}$ & $0.007^{* * * \pi}$ & $0.007^{* * * \pi}$ & $0.007^{* * \pi}$ & $0.007^{* * * * *}$ \\
\hline IndDirPct & -1.955 & -1.436 & -0.105 & -0.079 & -0.105 & -0.079 \\
\hline Gender & $-2.669^{*}$ & -2.370 & $-0.132^{*}$ & $-0.122^{*}$ & $-0.131^{*}$ & $-0.122^{* * * *}$ \\
\hline Salary & $-0.903^{* \prime \prime}$ & -0.562 & $-0.050^{n+m}$ & -0.032 & $-0.050^{\pi}$ & $-0.032^{2}$ \\
\hline ROA & 0.287 & 0.380 & -0.009 & -0.003 & -0.009 & -0.003 \\
\hline Netgrowth & $-0.579^{* * *}$ & $-0.563^{* * 2 \times}$ & $-0.027^{* *}$ & $-0.026^{* * \pi}$ & $-0.027^{* * *}$ & $-0.026^{* * * *}$ \\
\hline OwnCon5 & $-6.390^{* * \pi}$ & $-6.651^{* * 2 \times 1}$ & $-0.271^{4 * 2 \times 4}$ & $-0.286^{4 * 2 \times 4}$ & $-0.271^{3 * 2 \times}$ & $-0.286^{* * * *}$ \\
\hline Size & $6.459^{\text {**** }}$ & $6.326^{\text {*** }}$ & $0.284^{* * *}$ & $0.277^{* * *}$ & $0.284^{* * *}$ & $0.277^{* * *}$ \\
\hline Year & & Yes & & Yes & & Yes \\
\hline Industry & & Yes & & Yes & & Yes \\
\hline F值 & $251.68^{* \pi \times 4}$ & $154.42^{* 2 \times n}$ & $191.38^{* *}$ & $116.74^{* *}$ & $191.32^{\text {* }}$ & $116.71^{* * * \pi}$ \\
\hline $\mathrm{N}$ & 5008 & 5008 & 5008 & 5008 & 5008 & 5008 \\
\hline Adj- $R^{2}$ & 0.3106 & 0.3153 & 0.2550 & 0.2595 & 0.2549 & 0.2595 \\
\hline
\end{tabular}

注: $*$, **, ***分别表示在 $10 \%, 5 \%, 1 \%$ 的水平上显著

进一步研究高管性别差异对外部融资 方式的偏好, 分别将债权融资总额与发行总 股数和流通股股数的比值作为因变量进行 回归。由表7可知, 高管性别的回归系数均 为负数且在 $1 \%$ 的水平上显著, 即高管性别与 公司的债权股权比呈显著的负相关关系, 说 明相比于男性高管, 女性高管在选择外部融 资方式时, 更加倾向于选股权融资, 从而降 低公司的债权股权比例。此外, 董事会规模 和公司规模会显著增加公司的外部融资和 债权股权比, 而管理者平均薪酬、净资产增 长率及前五大股东持股比例则会明显降低 公司的外部融资和债权股权比。

综上, 高管性别差异与上市公司融资偏 好的回归结果也只是部分支持假设二, 即在 制定融资决策时，董事长、 $\mathrm{CE} 0$ 和CFO中含有 女性成员的公司会优先选择内部融资从而 减少外部融资, 在选择外部融资方式时又更 加倾向于选择股权融资而非债权融资, 这与 一些学者认为中国上市公司的融资行为存 在显著的股权融资偏好（黄少安、张岗, 2001) 的结论相一致。本文的结论并不完全 支持女性高管相比于男性高管更不容易表 现出过度自信的理论。

\section{4稳健性检验}

本文做了如下稳健性检验：（1）变更 投资效率的衡量方法。上文是用Richardson （2006）模型的残差来衡量公司的投资效 率, 在稳健性检验中采用Biddle（2009）的
模型衡量公司的投资效率, 得出的回归结果 与上文关于投资效率部分的结论基本一致。

（2）变更高管性别的衡量方法。将高管性 别的衡量指标改为女性高管的人数, 即若董 事长、CE0及CF0中有一人为女性，则高管性 别变量为 1 ; 有两人为女性, 则为 2 ; 三人为 女性则为 3 。回归分析的结果也与上文结论 基本一致。因此, 可以认为本文的研究结论 具有较强的稳健性。

\section{5. 结束语}

本文研究结果表明: 高管性别对公司投 资效率和并购决策的影响并不明显, 但董事 长、CEO和CFO中含有女性成员的上市公司 会显著减缓公司规模的增长速度, 也会显著 降低公司的外部融资规模, 在选择外部融资 方式时, 存在女性高管的上市公司更倾向于 选择股权融资, 从而降低公司的债股比例。

希望本文的研究能提高对高管团队性 别结构的重视, 将性别差异与投融资决策的 关系纳入聘用公司高管人员的考察范围内, 摒弃性别歧视, 让女性能在公司治理领域发 挥其应有的作用, 并最终提高公司价值。

\section{References}

[1] Zuckerman, M., Behavioral expressions and biosocial bases of sensation seeking. Personality and Individual Differences, vol.18, pp. 813-814, 1995. 
[2] Jiekun Huang, Darren J. Kisgen, Gender and corporate finance: Are male executives overconfident relative to female executives, Journal of Financial Economics, vol.108, pp. 822-839, 2013.

[3] Maurice Levia, Kai Li, Feng Zhang, Director Gender and Mergers and Acquisitions, Journal of Corporate Finance, vol.18, pp. 185-200, 2014.

[4] Barber, B.\& Odean, T., Boys will be boys: Gender, overconfidence, and common stock investment, Quarterly Journal of Economics, vol.116, pp. 261-293, 2001.

[5] ZHAO Bing-mei, WU Hui-min, The Analysis of the Effect of the Gender Differences of Executives on the Listed Company Investment, Value Engineering, vol.19, pp. 148-150, 2013.

[6] Graham, J., Harvey, C., Puri, M, Managerial attitudes and corporate Actions, Journal of Financial Economics, vol.109, pp. 103-121, 2013.

[7] LI Shi-gang, Female Managers, Overconfidence and Financing Preference, Economic Survey, vol.31, pp. 92-96, 2014. 